\title{
Electron-induced chemistry of alcohols
}

\author{
Bogdan C. Ibănescu, Olivier May, Angèle Monney and Michael Allan*
}

We studied dissociative electron attachment to a series of compounds with one or two hydroxyl groups. For the monoalcohols we found, apart from the known fragmentations in the 6-12 eV range proceeding via Feshbach resonances, also new weaker processes at lower energies, around $3 \mathrm{eV}$. They have a steep onset at the dissociation threshold and show a dramatic $\mathrm{D} / \mathrm{H}$ isotope effect. We assigned them as proceeding via shape resonances with temporary occupation of $\sigma_{\mathrm{O}-\mathrm{H}}^{*}$ orbitals. These low energy fragmentations become much stronger in the larger molecules and the strongest DEA process in the compounds with two hydroxyl groups, which thus represent an intermediate case between the behavior of small alcohols and the sugar ribose which was discovered to have strong DEA fragmentations near zero electron energy [S. Ptasińska, S. Denifl, P. Scheier and T. D. Märk, J. Chem. Phys., 2004, 120, 8505]. Above $6 \mathrm{eV}$, in the Feshbach resonance regime, the dominant process is a fast loss of a hydrogen atom from the hydroxyl group. In some cases the resulting $(M-1)^{-}$anion (loss of hydrogen atom) is sufficiently energyrich to further dissociate by loss of stable, closed shell molecules like $\mathrm{H}_{2}$ or ethene. The fast primary process is state- and site selective in several cases, the negative ion states with a hole in the $n_{\mathrm{O}}$ orbital losing the $\mathrm{OH}$ hydrogen, those with a hole in the $\sigma_{\mathrm{C}-\mathrm{H}}$ orbitals the alkyl hydrogen.

\section{Introduction}

The interest in electron-induced chemical processes has recently been renewed by the discovery that electrons at subionization $^{1}$ and even subexcitation energies ${ }^{2}$ damage DNA and by the emerging applications in materials science. ${ }^{3}$ Electron-induced chemistry at subionization energies can proceed either via electronic excitation of the target or, particularly at energies too low for electronic excitation, by way of dissociative electron attachment (DEA). ${ }^{4}$ The latter mechanism is particularly important because even a high energy primary electron (or $\gamma$ photon) impinging on a material or on a biological tissue leads to a "shower" of low energy electrons, providing a large amount of subionization electrons with energies suitable for DEA.

These applications renewed the interest in the corresponding elementary step, the DEA to isolated polyatomic molecules in the gas phase and, in particular, to molecules of biological interest with many interesting findings. ${ }^{5-10}$ In our earlier work we studied several saturated compounds containing the amino- and the hydroxyl groups which could be considered as model compounds for biomolecules. ${ }^{11}$ We used the comparison between the HeI photoelectron spectra and the DEA spectra to identify the Feshbach resonances and to assign the DEA bands and found that all DEA bands could be explained by Feshbach resonances, the low-lying ones being localized on the functional groups. In the present paper we substantially extend the work on the alcohols and report several new

Department of Chemistry, University of Fribourg, Chemin du Musée 9, CH-1700 Fribourg, Switzerland

$\dagger$ The HTML version of this article has been enhanced with colour images. findings, in particular, the involvement of shape resonances at low energies, dramatic isotope effects, cases of state and site selective chemistry, and more insight into the mechanism of fragmentation of Feshbach resonances.

The process of DEA has been extensively studied in the past. ${ }^{4,12}$ As has been summarized in our earlier paper, ${ }^{11}$ many DEA spectra of polyatomic molecules follow the same general pattern. They can be divided into a low energy domain, between zero and about $\sim 5 \mathrm{eV}$, and a high energy domain, about 5-14 eV.

DEA in the low energy range is generally initiated by an electron capture to form a shape ('one particle') resonance. The initial phase of the DEA is characterized by a competition of a very fast autodetachment and stabilization of the negative ion by a distortion of the molecular frame. The bands in the low energy range can often be assigned by their coincidence in energy with resonances found in the electron transmission spectrum (ETS) or vibrational excitation cross sections. Insight into the mechanism can be gained by comparing the appearance energies of the various fragments with the thermochemical thresholds because generally only few dissociation channels are energetically open. The low energy processes in methyl acetate may serve as typical examples. ${ }^{13}$ In addition, vibrational Feshbach resonances (VFR) ${ }^{14}$ may serve as doorway states to initiate DEA at very low energies. Representative examples are $\mathrm{CH}_{3} \mathrm{I},{ }^{15} \mathrm{~N}_{2} \mathrm{O}^{16}$ and ethylene carbonate. ${ }^{17}$ The VFR mechanism has been shown to be operative in uracil and thymine. .,18 $^{-18}$

In virtually all compounds one or several DEA bands are also found in the 5-14 eV range. The assignment of these bands is more difficult because no (or only very weak) corresponding features are found in the other channels of electron scattering, like vibrational excitation or total crosssection (as revealed by the ETS). The situation is complicated 
by the fact that dissociation into many different fragments is energetically possible, and many different fragments often actually occur. It appears that most DEA bands in this energy range are due to Feshbach resonances with double occupation of Rydberg-like orbitals and a positive ion core. ${ }^{19,20}$

An unambiguous assignment of the high energy bands to such resonances has occasionally been possible in small molecules where the DEA bands have sharp vibrational structure which resembles that of a Rydberg excited state of the neutral molecule (the parent state) and that of the photoelectron band of the positive ion (the grandparent state). Examples are ammonia $^{20}$ and acetaldehyde. ${ }^{21,22}$ In view of the high energy and the competition with fast autodetachment it is somewhat surprising that complex fragmentations involving 'scrambling' are often observed. They indicate that a relatively long time is available for the dissociation. The mechanism involves propagation of the nuclear wave packet on a manifold of the Rydberg-like anion states, possibly predissociated by repulsive valence states, as recently explicitly calculated for water. ${ }^{23}$ The analogy to conical intersection ${ }^{24,25}$ is involved.

Feshbach resonances with occupation of Rydberg-like orbitals are well known from scattering experiments other than DEA, in particular ETS of rare gases, diatomic and polyatomic molecules ${ }^{26-29}$ or in the vibrational excitation crosssections (for an example see ref. 30). The energies of these Feshbach resonances were found to have a simple relation to the energies of the parent Rydberg states and the grandparent state of the cation. The relation depends only weakly on the molecule in question. The electron affinity of the parent Rydberg state is of the order of $0.3-0.5 \mathrm{eV}$ for the $s^{2}$ configuration. $^{26-29,31}$ Read expressed the relation with a modified Rydberg-Ritz formula. ${ }^{32}$ Spence $^{33}$ fitted a linear relationship between the energy of the Feshbach resonance $E_{F}$ and the first ionization energy $I, E_{F}=A \cdot I+B$, for many hydrogen- and methyl halides. The slope $A$ had the value of around 1. The constant $B$ had the values -3.9 and $-1.8 \mathrm{eV}$ for the $s^{2}$ and $p^{2}$ configurations, respectively. This means that the $s^{2}$ Feshbach resonance was always found $\sim 4 \mathrm{eV}$ below its grandparent state, the ground state of the ion.

For completeness, it should be mentioned that in an intermediate energy range, $\sim 4 \mathrm{eV}$, DEA bands have been found which have nearly the same energy as, and resemble in shape, the low-lying singlet valence excited states of the target molecule. These bands have been found in unsaturated compounds and assigned to resonances where an $s$-like electron is weakly bound to a valence-excited core. Examples are $\mathrm{CS}_{2}{ }^{34}$ and other molecules (for example, norbornadiene, cyclopentadiene, $\left.\mathrm{SO}_{2}\right){ }^{35}$

This paper presents dissociative electron attachment spectra of a series of alcohols and attempts to assign the observed bands to various types of resonances as outlined above. The assignment is guided by a comparison with HeI photoelectron spectra and vibrational excitation cross-sections.

\section{Methods}

The dissociative electron attachment spectrometer used to measure the yield of mass-selected stable anions as a function of electron energy has been described previously. ${ }^{17,36}$ It employs a magnetically collimated trochoidal electron monochromator to prepare a beam of quasi-monoenergetic electrons which is directed into a target chamber filled with a quasi-static sample gas. Fragment anions are extracted at $90^{\circ}$ by a three-cylinder lens and directed into a quadrupole mass spectrometer. The energy scale was calibrated on the onset of the $\mathrm{O}^{-} / \mathrm{CO}_{2}$ signal at $4.0 \mathrm{eV}$. The electron current was several hundreds of nanoamperes and the resolution about $150 \mathrm{meV}$.

A common problem with DEA instruments equipped with a trochoidal monochromator is the difficulty of detecting light ions, specifically $\mathrm{H}^{-}$and $\mathrm{D}^{-}$, because the magnetic field required for the operation of the trochoidal monochromator is perpendicular to the ion path and deviates the light ions in the ion transporting lens, preventing them from reaching the quadrupole mass spectrometer. (The time-of-flight spectrometer without a magnetic field of Krishnakumar is better in this respect. ${ }^{10}$ ) Our ion lens has two pairs of $x, y$ deflectors, situated near its entrance and its exit, ${ }^{17}$ which were used to compensate the deflection by the magnetic field. The electron beam current and, consequently, the sensitivity have been improved in comparison to our earlier work, permitting the detection of weak bands not reported earlier. Care was taken to make the measurements at sufficiently low pressures as ion-molecule reactions of the type

$$
\mathrm{O}^{-}+\mathrm{ROH} \rightarrow \mathrm{RO}^{-}+\mathrm{OH}
$$

were often found to distort the spectra at higher pressures.

The vibrational excitation cross-sections were recorded with a spectrometer using hemispherical electron-energy selectors. ${ }^{37}$ The photoelectron spectra were recorded with a modified Perkin Elmer PS18 HeI photoelectron spectrometer.

Threshold energies for various fragmentations were calculated as the differences of the total energies of the products and the targets at $0 \mathrm{~K}$, corrected for the zero point vibrational energy, using the density functional theory (DFT) B3LYP/6$311+\mathrm{G}(2 \mathrm{df}, 2 \mathrm{p})$ model. $^{38}$

\section{Results and discussion}

The alcohols studied in this work are shown in the following scheme (Scheme 1):

They include the two simple alcohols methanol and ethanol, and a series of alcohols all of which are to some degree related

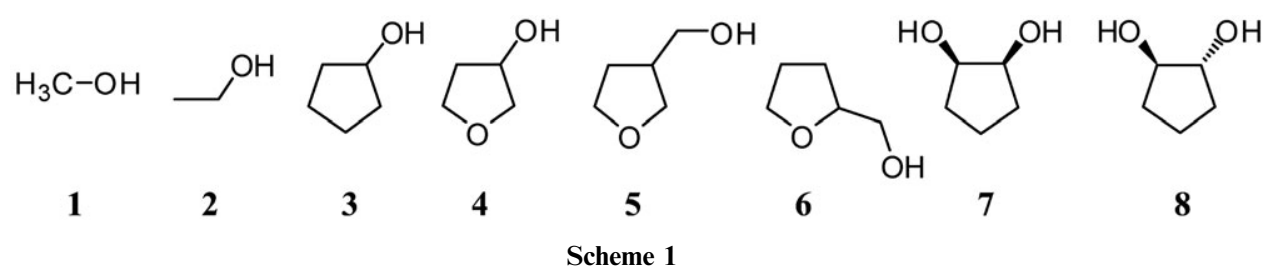




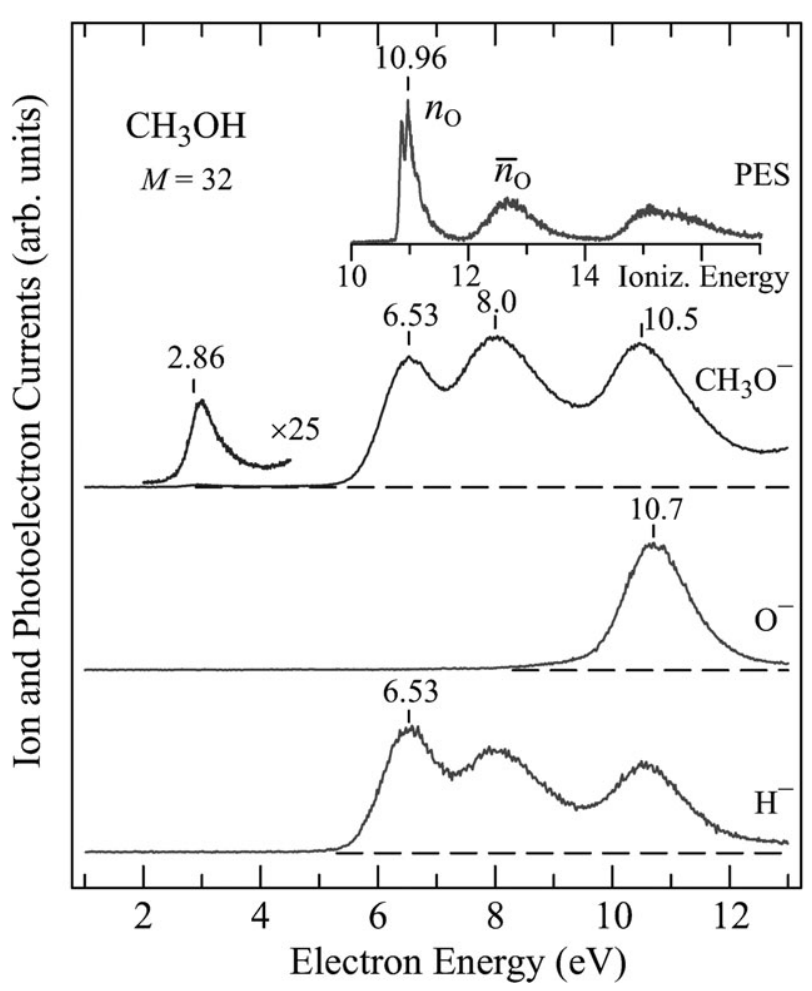

Fig. 1 The HeI photoelectron spectrum (top, shown shifted by $-4.5 \mathrm{eV})$ and the yields of the $(M-1)^{-}, \mathrm{H}^{-}$and $\mathrm{O}^{-}$fragments from methanol (1).

to tetrahydrofuran and ribose, which have been of interest recently ${ }^{9,39,40}$ because of their relevance for DNA.

The figures shown in this paper generally compare the photoelectron spectra, which have been found important for identifying Feshbach resonances, with the yield of the $(M-$ $1)^{-}$fragment (loss of hydrogen atom), or $(M-2)^{-}$(loss of deuterium) - often the primary fragments in alcohols. ( $M$ is the molecular mass and $(M-1)^{-}$stands for an anion with a mass of $M-1$.) The complementary fragment, $\mathrm{H}^{-}$or $\mathrm{D}^{-}$, and selected fragments considered interesting for the discussion are also shown. Fragments judged less essential for the present work are not shown but are mentioned in the text.

\subsection{Methanol (1)}

Kühn et al. ${ }^{41}$ studied dissociative electron attachment to methanol including fully and partially deuterated isotopomers. They also analyzed the translational energies of the ionic fragments. They observed $\mathrm{CH}_{3} \mathrm{O}^{-}, \mathrm{OH}^{-}$and $\mathrm{O}^{-}$fragments in three bands in the 5-12 eV range which they assigned to core excited resonances with two electrons in a Rydberg-like MO. The observation of unexpected fragments from the isotopically substituted compounds, i.e., $\mathrm{OH}^{-} / \mathrm{CH}_{3} \mathrm{OD}$ or $\mathrm{OD}^{-} / \mathrm{CD}_{3} \mathrm{OH}$ revealed hydrogen scrambling for these two fragments, in contrast to the formation of $\mathrm{CH}_{3} \mathrm{O}^{-}$(and its deuterated analogue), which proceeded without scrambling. Methanol has also been studied by Curtis and Walker, ${ }^{42}$ who discovered a substantial kinetic energy release for $\mathrm{H}^{-}$and compared the Feshbach resonances in the DEA spectra to the parent Rydberg states in the VUV spectrum. They also
Table 1 Threshold energies for the reactions $\mathrm{e}^{-}+\mathrm{ROH} \rightarrow \mathrm{RO}^{-}+$ $\mathrm{H}$, in eV. The DFT values were calculated as described in section 2 . The "therm." values were derived from experimental thermochemical data, in particular the gas phase acidities. ${ }^{43}$ The onsets of the DEA bands were taken from the present spectra

\begin{tabular}{llll}
\hline \multirow{2}{*}{ Target } & $E_{\mathrm{thr}}$ & & \\
\cline { 2 - 4 } & DFT & Therm. & Onset of DEA \\
\hline $\mathbf{1}$ & 2.82 & 2.94 & 2.86 \\
$\mathbf{2}$ & 2.69 & 2.81 & 2.75 \\
$\mathbf{3}$ & 2.53 & & 2.67 \\
$\mathbf{4}$ & 2.31 & & 2.50 \\
$\mathbf{5}$ & 2.35 & & 2.36 \\
$\mathbf{6}$ & 2.64 & & 2.58 \\
$\mathbf{7}$ & 1.89 & & 2.94 \\
$\mathbf{8}$ & 2.43 & & 2.43 \\
\hline
\end{tabular}

observed an early indication of site selectivity $-\mathrm{D}^{-}$originated from the OD group at 5-8 eV, but from both hydroxyl and methyl groups around $10.5 \mathrm{eV}$. The concept of site selectivity was extended to other compounds and generalized by Prabhudesai et al. ${ }^{10}$

Our current spectrum of the $\mathrm{CH}_{3} \mathrm{O}^{-}$fragment, shown in Fig. 1, is consistent with the earlier spectra but additionally reveals a weaker band peaking at $2.99 \mathrm{eV}$. Its left side is steep, consistent with a vertical onset at the energetic threshold, calculated from the experimental methanol acidity of $1596 \mathrm{~kJ} \mathrm{~mol}^{-1} 43$ to be $2.94 \mathrm{eV}$ (Table 1). Since we did not find the required experimental acidities of all the compounds measured in this work, we also used DFT calculations to determine the threshold energies. Comparison of the threshold energies determined from experimental thermochemical data and calculated using quantum chemical methods for methanol and ethanol (Table 1) indicates that the latter is reliable within about $\pm 0.2 \mathrm{eV}$, sufficient for the present purposes. The observed DEA bands are broadened by the finite resolution of the experiment and possibly also by 'hot bands', signals from thermally vibrationally excited molecules. It is therefore not quite trivial to decide at what position on the observed band the experimental onset should be taken-it depends on the inherent shape of the band. For a step-shaped band the onset should be taken at $50 \%$, for a line-shaped band at $100 \%$ of the band height. Since the real band shape is somewhere between these two extremes we adopted the same pragmatic approach as in an earlier work ${ }^{44}$ and take the value at $75 \%$ of the height. The agreement between calculation and theory obtained with these rules and indicated in Table 1 is satisfactory.

The $2.99 \mathrm{eV}$ band can not be assigned to a core-excited resonance because of lack of suitable parent electronically excited states (both Rydberg and valence). At the same time the energy is too high for an assignment to a dipole-bound resonance or a virtual state. The dissociation must thus proceed via a ${ }^{2}\left(\sigma^{*}\right)$ shape resonance, as will be discussed in more detail below in connection with ethanol. The $\mathrm{H}^{-}$yield, also shown in Fig. 1, is very similar in shape to the $\mathrm{CH}_{3} \mathrm{O}^{-}$ yield above $6 \mathrm{eV}$, indicating that the same Feshbach resonances dissociate into both fragments - the charge distribution is not selective. The $2.99 \mathrm{eV}$ band is missing in the $\mathrm{H}^{-}$ yield - this is probably because of the higher threshold for $\mathrm{H}^{-}$formation. The $\mathrm{O}^{-}$yield is consistent with earlier results. ${ }^{41}$ 


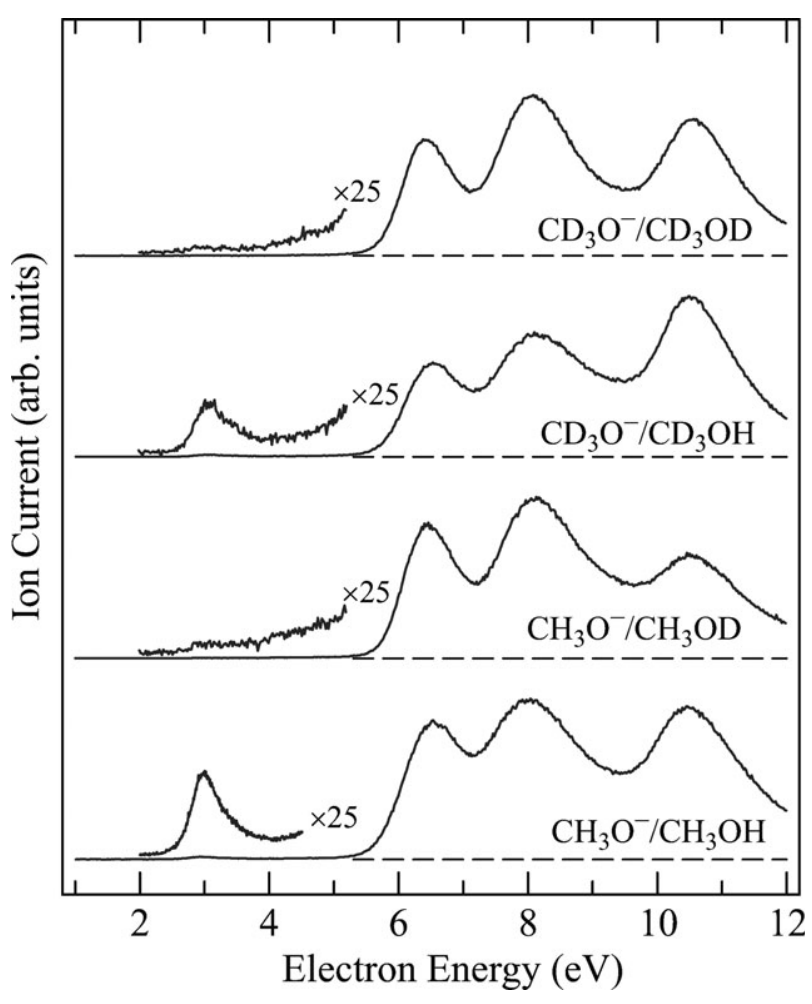

Fig. 2 Yield of the methanolate anion fragment from various isotopically substituted methanols.

Study of the fully and the partially deuterated methanols revealed a strong isotope effect for the $3 \mathrm{eV}$ band (Fig. 2). The signal has dropped below the detectability limit when the hydroxyl group was deuterated, indicating an isotope effect of more than a factor of 10 . The process thus resembles the formation of $\mathrm{H}^{-}$from $\mathrm{H}_{2}$ where the large width of the ${ }^{2} \Sigma_{\mathrm{u}} \sigma^{*}$ shape resonance $\left(\Gamma \sim 4 \mathrm{eV}\right.$ at the $\mathrm{H}_{2}$ equilibrium internuclear distance in the nonlocal model of Mündel et al. ${ }^{45}$ ) causes an isotope effect of a factor of $200 .{ }^{46}$ Physically, the dramatic drop of the cross-section is a consequence of the slower motion of the heavier deuterium compared with the lighter hydrogen, leading to longer dissociation time, and thus less favorable competition of dissociation with the very fast autodetachment.

Our present experiment does not measure absolute crosssections but we estimated the relative cross-sections for the various methanol isotopomers by recording signal intensities for the same pressure (same Penning gauge reading) and the same electron beam current. These measurements confirmed the strong isotope effect for the $3 \mathrm{eV}$ band mentioned above where the cross-section for breaking the $\mathrm{O}-\mathrm{H}$ bond is at least $10 \times$ larger than that for breaking the O-D bond. Deuteration of the methyl group also has an effect, albeit weaker, on the cross-section for breaking the $\mathrm{O}-\mathrm{H}$ bond - the cross-section for breaking the $\mathrm{O}-\mathrm{H}$ bond in $\mathrm{CD}_{3} \mathrm{OH}$ appears to be about $2 \times$ larger than the cross-section for breaking the $\mathrm{O}-\mathrm{H}$ bond in $\mathrm{CH}_{3} \mathrm{OH}$. This observation is somewhat surprising because the above argument is not applicable - the $\mathrm{O}-\mathrm{H}$ bond would be expected to dissociate equally fast whether the methyl group is deuterated or not. The reason could be related to the fact that the $\mathrm{C}-\mathrm{H}$ modes in the methyl-deuterated compound are, in terms of frequency, better separated from the dissociating

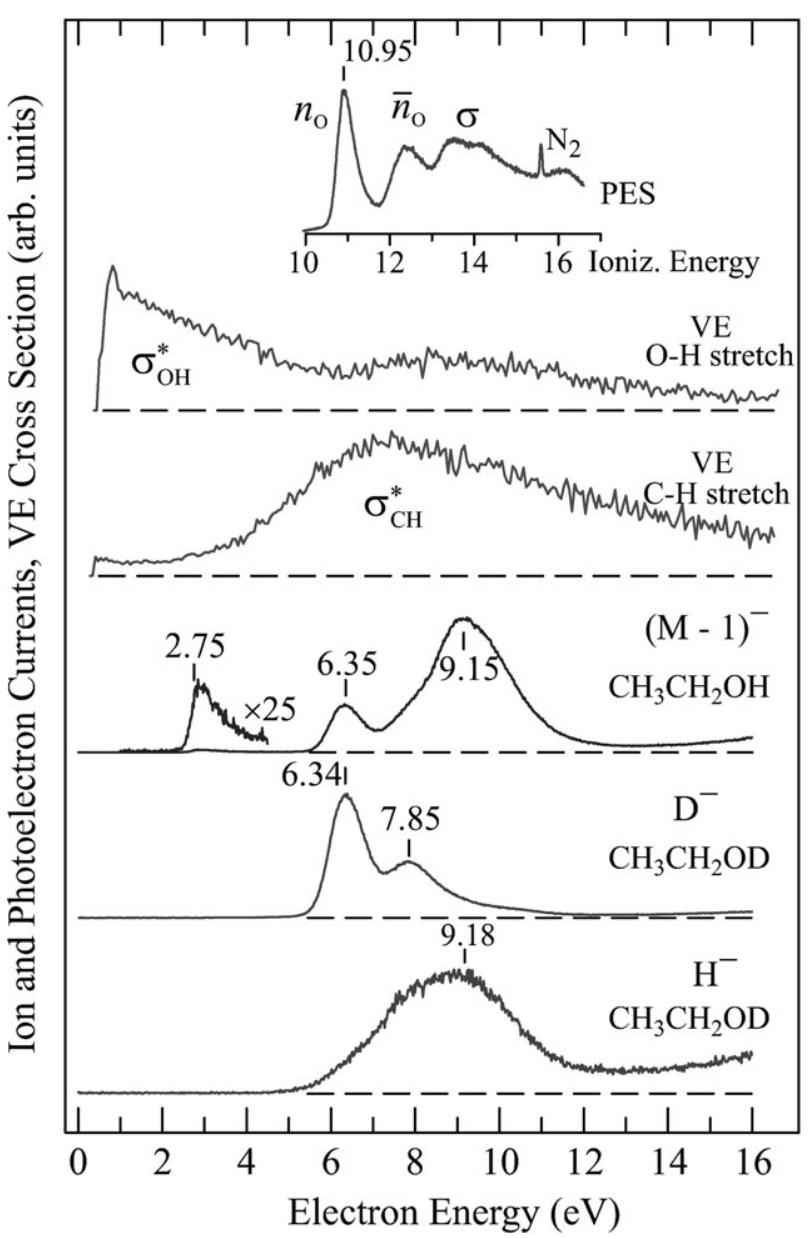

Fig. 3 Comparison of selected DEA spectra with vibrational excitation cross-sections (measured at $135^{\circ}$ ) and photoelectron spectra for ethanol (2). The PE spectrum is shown shifted by $-4.65 \mathrm{eV}$; a weak sharp peak due to a $\mathrm{N}_{2}$ impurity is marked. $\mathrm{D}^{-}$and $\mathrm{H}^{-}$yields from ethanol- $\mathrm{d}_{1}$ are shown in the bottom two spectra.

(O-H stretch) mode, reducing the possibility of fast intramolecular vibrational redistribution (IVR) which would quench the dissociation.

The pressure-normalized measurements further revealed (weaker) isotope effects for the bands in the Feshbach resonance region. The relative intensities of the three methanolate bands in the 6-12 eV range (i.e., the shape of the spectrum in this energy range) also depend to some degree on deuteration (Fig. 1 and 2). The intensity of the highest band $(8.1 \mathrm{eV})$ increased with deuteration, being about $2 \times$ larger in $\mathrm{CD}_{3} \mathrm{OD}$ than in $\mathrm{CH}_{3} \mathrm{OH}$. $\mathrm{CH}_{3} \mathrm{OD}$ yielded also the $\mathrm{CHO}^{-}$fragment (loss of $\mathrm{D}$ and of $\mathrm{H}_{2}$ ).

\subsection{Ethanol (2)}

Fig. 3 shows the spectra for the major fragments from ethanol. The $6.35 \mathrm{eV}$ band in the $(M-1)^{-} / \mathrm{EtOH}$ and $\mathrm{D}^{-} / \mathrm{EtOD}$ spectra can be assigned to a Feshbach resonance with a hole in the $n_{\mathrm{O}}$ oxygen lone pair orbital. The Feshbach resonance with a hole in the $\bar{n}_{\mathrm{O}}$ oxygen lone pair orbital appears as a weaker peak at $7.85 \mathrm{eV}$ in the $\mathrm{D}^{-} /$EtOD spectrum and as an indistinct shoulder at the same energy in the $(M-1)^{-} /$EtOH spectrum. 


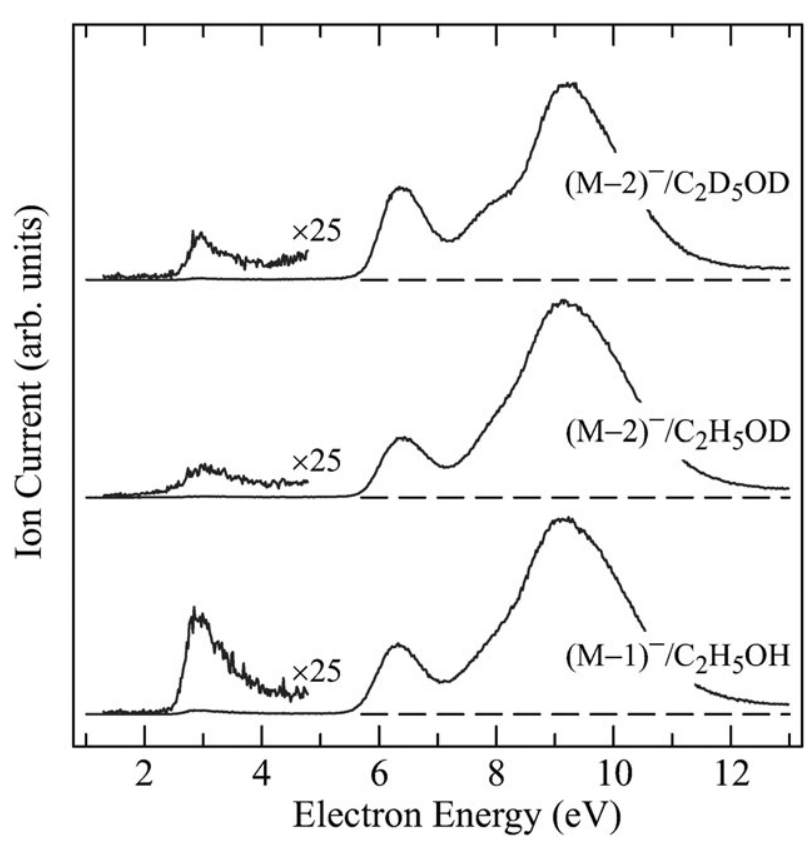

Fig. 4 Yield of the ethanolate anion fragment from various isotopically substituted ethanols.

Finally the photoelectron spectrum shows overlapping bands due to ionization from various $\mathrm{C}-\mathrm{H}$ and $\mathrm{C}-\mathrm{C} \sigma$ orbitals above $13 \mathrm{eV}$ and the broad DA bands appearing around $9.15 \mathrm{eV}$ in the DA spectra can be assigned to the corresponding Feshbach resonances.

A weaker band appears at $2.88 \mathrm{eV}$ in the $(M-1)^{-} / \mathrm{EtOH}$ spectrum. It can be assigned to a very short-lived $\sigma_{\mathrm{OH}}^{*}$ shape resonance, similarly to methanol. The shape of the band is asymmetrical, consistent with a vertical onset at the energetic threshold. The signal onset, taken at $75 \%$ of peak height, is at $2.75 \mathrm{eV}$ in good agreement both with the value of $2.81 \mathrm{eV}$ determined from the experimental gas phase acidity of ethanol $^{43}$ and the value of $2.69 \mathrm{eV}$ obtained from DFT calculations (Table 1). As in the methanol case, a very short lifetime (large width) of the resonance responsible for the $2.88 \mathrm{eV}$ band is deduced from the very pronounced isotope effect, shown in Fig. 4: the band is about $4 \times$ weaker when the hydroxyl hydrogen is replaced by deuterium.

Additional evidence for the identity of resonance processes is provided by the cross-sections for vibrational excitation (VE) because they are generally enhanced by resonances, in particular by shape resonances ${ }^{47}$ and by vibrational Feshbach resonances. ${ }^{14}$ We therefore compare the DEA spectra to selected VE spectra in Fig. 3. The cross-sections for the excitation of two vibrations are shown. The spectrum for the excitation of the $\mathrm{C}-\mathrm{H}$ stretch vibration is similar to the $\mathrm{C}-\mathrm{H}$ stretch excitation in hydrocarbons ${ }^{48}$-it consists of a very broad band peaking around $7.5 \mathrm{eV}$ which could be assigned to a temporary occupation of $\sigma_{\mathrm{CH}}^{*}$ orbitals, the large width of the band indicating a very large autodetachment width and possibly also several overlapping resonances.

The cross-section for the excitation of the $\mathrm{O}-\mathrm{H}$ stretch vibration is similar to the cross-section observed in formic acid $^{49}$ - it peaks at threshold and then decreases slowly over an energy range of several $\mathrm{eV}$. The electron cloud associated with this resonant process must be located on the $\mathrm{O}-\mathrm{H}$ bond (because it excites selectively the $\mathrm{O}-\mathrm{H}$ vibration). The band in the $\mathrm{O}-\mathrm{H}$ stretch vibrational excitation cross-section is much broader than the band in the $(M-1)^{-}$DEA band in Fig. 3, but we propose that both processes are due to the same $\sigma^{*}$ shape resonance, possibly enhanced by dipole binding at low energies. It is well established that shape resonances with large autodetachment widths can result in relatively narrow DEA bands. The prototype examples are $\mathrm{H}_{2}{ }^{50,51}$ and the halogen halides. ${ }^{14,52}$ The $\mathrm{H}_{2}$ case has in common with the present observation in ethanol also the large isotope effect. The cause of the striking difference of the DEA and the VE band widths in these cases is, on the one hand, the vertical DEA signal rise at the threshold and, on the other hand, the rapid increase of the resonant autodetachment width with energy ${ }^{50,51}$ and consequently rapid decrease of the DEA cross-section.

The hydrogen halide resonance phenomena at low energies are the result of a $\sigma^{*}$ valence state at large internuclear distances and dipole binding of the extra electron at shorter internuclear distances, ${ }^{14,52}$ which causes the anion potential curve to bend down before it crosses the potential curve of the neutral molecule. A similar situation has also been identified in uracil and thymine. ${ }^{18}$ The dipole moments of methanol (1.7 D) and ethanol (1.5 D) are not much less than that of HF (1.8 D) and it is thus probable that dipole binding also bends the anion potential surface down at stretched $\mathrm{O}-\mathrm{H}$ distances and

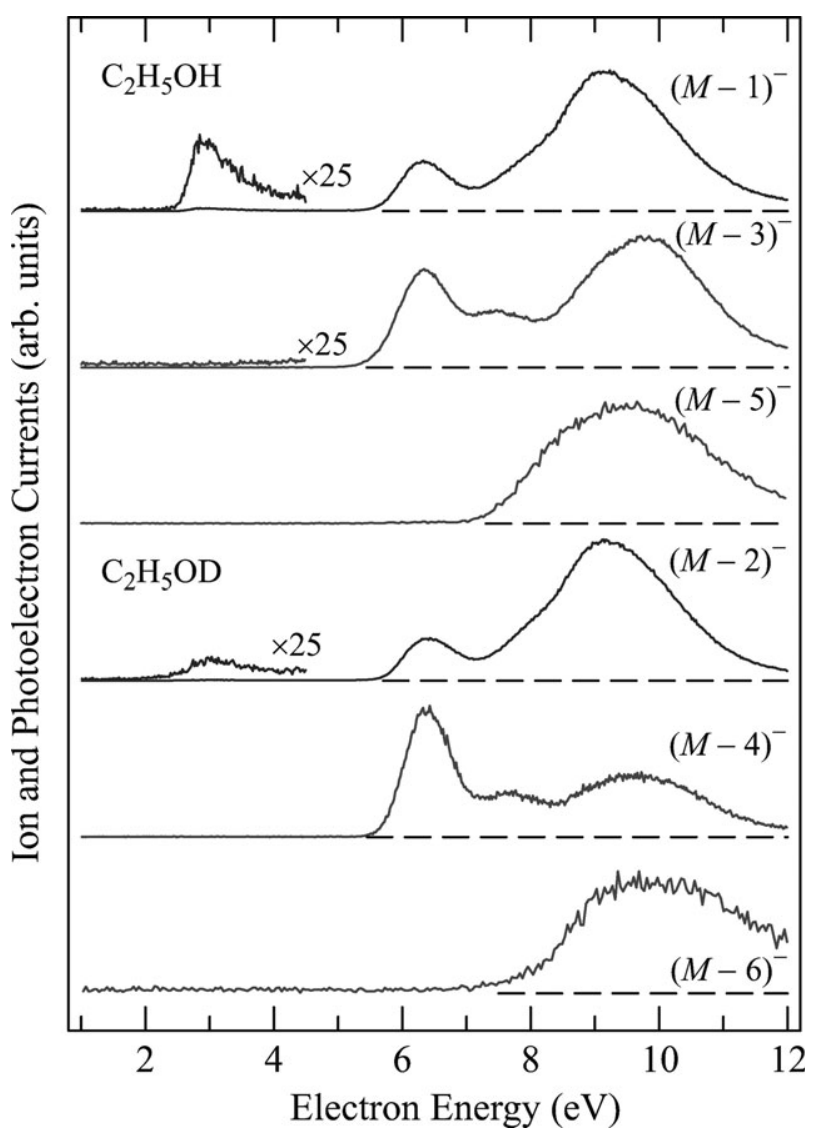

Fig. 5 Yields of fragments resulting from a loss of a hydrogen atom and subsequent losses of one and two hydrogen molecules. The top three spectra refer to $\mathrm{C}_{2} \mathrm{H}_{5} \mathrm{OH}$, the lower three spectra to $\mathrm{C}_{2} \mathrm{H}_{5} \mathrm{OD}$. 


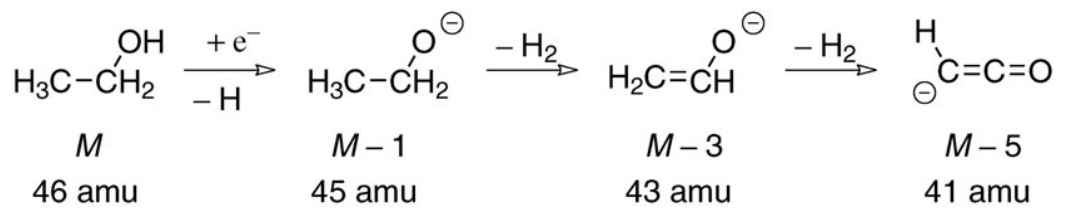

Fig. 6 Tentative reactions leading to fragments with masses $M-1, M-3$ and $M-5$ from ethanol (2).

affects VE and DEA in the alcohols at low energies. An ultimate proof of vibrational Feshbach resonances are the structures in the VE cross-sections slightly below the vibrational levels of the neutral molecules - like those found in $\mathrm{HF}$ or $\mathrm{CO}_{2} \cdot{ }^{14}$ We searched but did not find any such structures in methanol and ethanol VE cross-sections, however.

The DEA bands at 6.0, 7.7 and $8.8 \mathrm{eV}$ do not have any obvious analogs in VE (Fig. 3), providing a further evidence that they are due to core excited Feshbach resonances and not to $\sigma^{*}$ shape resonances.

Fig. 5 shows the yields of the less expected fragments $(M-3)^{-}$and $(M-5)^{-}$from EtOH and the corresponding fragments from EtOD. Fig. 6 shows tentative structures of the products and Table 2 gives the corresponding calculated threshold energies. The $(M-3)^{-}$fragment is presumably the result of a loss of an $\mathrm{H}$ atom and an $\mathrm{H}_{2}$ molecule and its structure could be that of an acetaldehyde deprotonated in the $\alpha$ position - the negative charge is stabilized by delocalization over three atoms which results in the relatively large acidity of the $\alpha$ hydrogen in carbonyl compounds. For this reason it has already been found to be an important fragment in DEA to acetaldehyde. ${ }^{53}$ Table 2 indicates that the threshold energy for this fragmentation is surprisingly low, in fact the fragmentation of the $\mathrm{CH}_{3} \mathrm{CH}_{2} \mathrm{O}^{-}$anion into $\mathrm{H}_{2}+\mathrm{CH}_{2} \mathrm{CHO}^{-}$is thermoneutral. This means that the $m / z=43$ fragment could appear already around $3 \mathrm{eV}$ - the fact that it is not observed there indicates that the fragmentation has an activation barrier and does not proceed without excess energy.

The $(M-5)^{-}$fragment is presumably the result of a loss of an $\mathrm{H}$ atom and two $\mathrm{H}_{2}$ molecules, and its structure could be that of a deprotonated ketene. Deprotonated ketene is a stable anion and has already been observed in DEA to methyl acetate. $^{44}$ The calculated threshold energy is well below the actual appearance of this fragment (Fig. 5).

Fig. 3 also illustrates another interesting effect-deuterium atom is lost from $\mathrm{CH}_{3} \mathrm{CH}_{2} \mathrm{OD}$ via the relatively narrow 6.35 and $7.85 \mathrm{eV}$ Feshbach resonances whereas hydrogen is lost via a broad $9.18 \mathrm{eV}$ feature. As already mentioned above, we

Table 2 Threshold energies for the more complex fragmentations, calculated as described in section 2. See Fig. 6 and 8 for tentative structures some of the polyatomic products

\begin{tabular}{llll}
\hline Target & Products & $m / z$ & $E_{\text {thr }}$ \\
\hline $\mathbf{2}$ & $\mathrm{H}+\mathrm{CH}_{3} \mathrm{CH}_{2} \mathrm{O}^{-}$ & 45 & 2.69 \\
& $\mathrm{H}+\mathrm{H}_{2}+\mathrm{CH}_{2} \mathrm{CHO}^{-}$ & 43 & 2.67 \\
\multirow{3}{*}{$\mathbf{3}$} & $\mathrm{H}+\mathrm{H}_{2}+\mathrm{H}_{2}+\mathrm{HCCO}^{-}$ & 41 & 3.72 \\
& $\mathrm{H}+c-\mathrm{C}_{5} \mathrm{H}_{9} \mathrm{O}^{-}$ & 85 & 2.53 \\
& $\mathrm{H}+\mathrm{H}_{2}+c-\mathrm{C}_{5} \mathrm{H}_{7} \mathrm{O}^{-}$ & 83 & 2.45 \\
\multirow{6}{*}{6} & $\mathrm{H}+\mathrm{CH}_{2}=\mathrm{CH}_{2}+\mathrm{CH}_{3} \mathrm{C}_{\left(\mathrm{CH}_{2}\right) \mathrm{O}^{-}}$ & 57 & 2.54 \\
& $\mathrm{H}+\mathrm{CH}_{3}-\mathrm{CH}_{3}+\mathrm{CH}_{2}=\mathrm{CHCOO}^{-}$ & 71 & 0.42 \\
& $\mathrm{H}_{2} \mathrm{C}-\mathrm{OH}+c-\mathrm{C}_{4} \mathrm{H}_{7} \mathrm{O}^{-}$ & 71 & 3.43 \\
\hline
\end{tabular}

assign this broad $9.18 \mathrm{eV}$ feature to Feshbach resonances with holes in the $\sigma_{\mathrm{CH}}$ orbitals, that is, we observe that hydrogens are lost from the hydroxyl group for Feshbach resonances located on the hydroxyl group, and hydrogens are lost from the alkyl group for Feshbach resonances located on the alkyl group. The broad $(M-1)^{-} 8.8 \mathrm{eV}$ band in the undeuterated molecule $\mathrm{CH}_{3} \mathrm{CH}_{2} \mathrm{OH}$ is the sum of both processes, in this case indistinguishable. The state selective chemistry observed here is similar to that reported recently in acetic acid by Krishnakumar. $^{10}$

\subsection{Cyclopentanol (3)}

The spectra are shown in Fig. 7. The larger alkyl group of cyclopentanol as compared to methanol or ethanol has the consequence of many more $\sigma_{\mathrm{C}-\mathrm{H}}$ and $\sigma_{\mathrm{C}-\mathrm{C}}$ orbitals and many more corresponding cation states which cause the "sigma

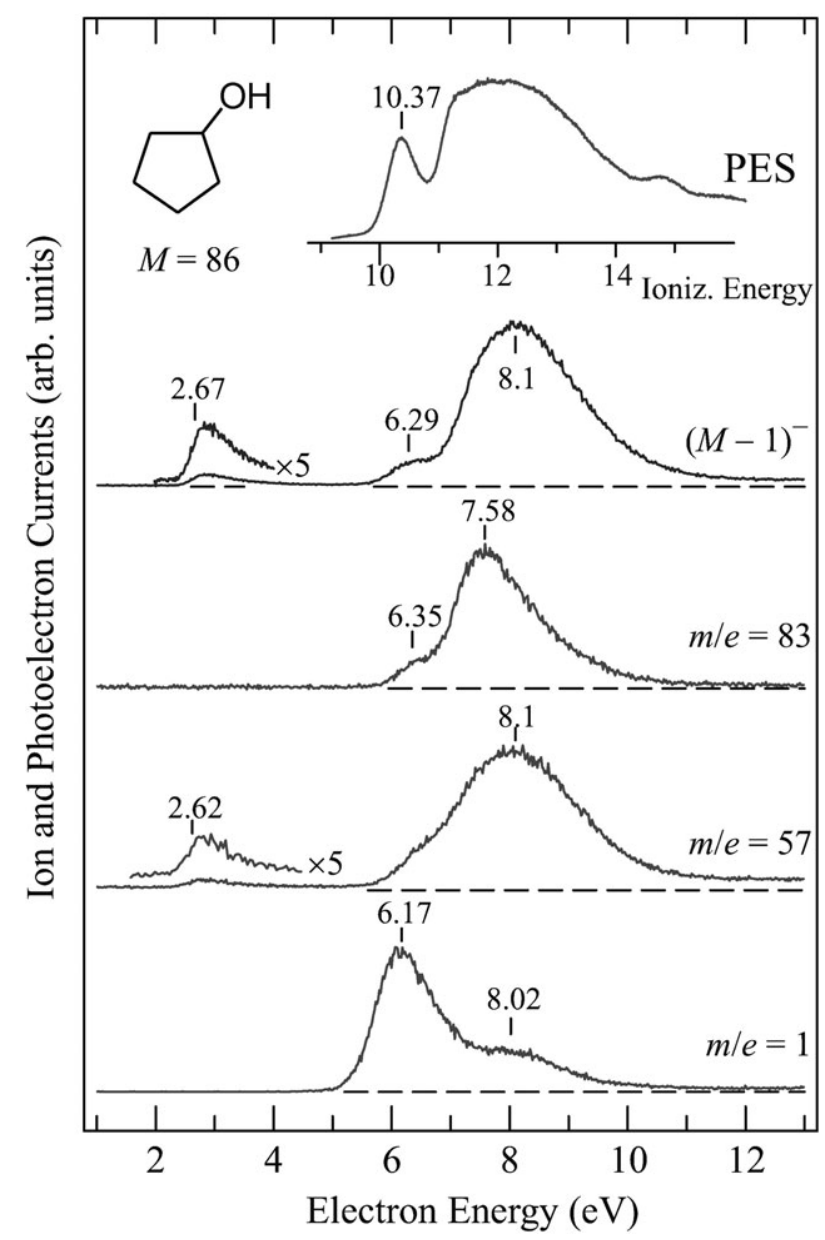

Fig. 7 Photoelectron and DEA spectra of cyclopentanol (3). The PE spectrum is shown shifted by $-4.25 \mathrm{eV}$. 


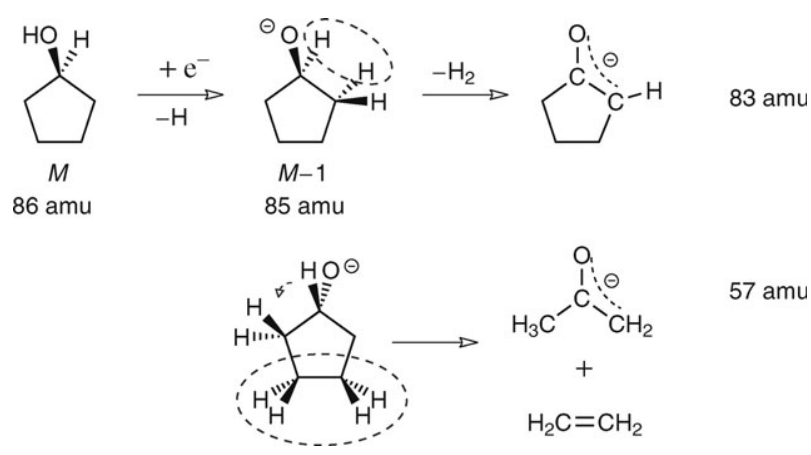

Fig. 8 Tentative equations leading to fragments with $m / z=57$ and 83 from cyclopentanol.

mountain" in the 11-14 eV range of the photoelectron spectrum. The first lone pair band $n_{\mathrm{O}}$ is visible as a separate band, but the second lone pair ionization $\bar{n}_{\mathrm{O}}$ overlaps with the $\sigma$ states. The $6.29 \mathrm{eV}$ shoulder in the $(M-1)^{-}$DEA spectrum can be assigned to a Feshbach resonance with a hole in the $n_{\mathrm{O}}$ lone pair orbital. The $7.58 \mathrm{eV}$ peak in the $m / z=83$ spectrum could be due to a Feshbach resonance with a hole in the $\bar{n}_{\mathrm{O}}$ lone pair orbital. The broad and unstructured band peaking around $8.1 \mathrm{eV}$ which appears in particular in the $(M-1)^{-}$and the $m / z=57$ spectra is assigned to several overlapping Feshbach resonances with $\sigma_{\mathrm{C}-\mathrm{H}}^{-1}$ and $\sigma_{\mathrm{C}-\mathrm{C}}^{-1}$ grandparent states.

DEA to cyclopentanol yields also the less expected fragments with masses with $m / z=57$ and 83 . The mass spectra do not, of course, give any direct evidence of their structures but in the scheme in Fig. 8 we show what we consider to be the most plausible candidates for these fragments. In both cases the short-lived resonance formed by electron attachment is first stabilized by the loss of hydrogen atom to form a cyclopentanolate anion with substantial vibrational energy. This anion can then lose an $\mathrm{H}_{2}$ molecule and form an $\alpha$ deprotonated cyclopentanone whose stability is the consequence of charge delocalization over three atoms and the ensuing relatively large acidity of $\alpha$-hydrogens in carbonyl compounds, as already encountered above in connection with the $\alpha$-deprotonated acetaldehyde fragment from ethanol. The pentanolate anion can also undergo an intramolecular hydrogen shift and lose an ethene molecule, yielding an $\alpha$-deprotonated acetone as shown in the lower part of Fig. 8. The loss of ethene appears to be a frequent process. In cyclobutanol $(m / z=72)$ we observed, apart from the $(M-1)^{-}$ion, only one prominent fragment which corresponds to the loss of ethene from the $(M-1)^{-}$ion, yielding an ion with $m / z=$ $43, \mathrm{H}_{2} \mathrm{C}=\mathrm{CHO}^{-}$, which can be understood as either the vinyl alcoholate ion or as an $\alpha$-deprotonated acetaldehyde.

Finally, the $2.67 \mathrm{eV}$ band in the $(M-1)^{-}$spectrum in Fig. 7 is assigned to dissociation via a shape resonance as in methanol and ethanol. The onset is at a slightly lower energy than in methanol, and this change is reflected in the calculated threshold energy (Table 1).

\subsection{Tetrahydrofuran-3-ol (4)}

The spectra are shown in Fig. 9. The photoelectron spectrum has a band at $9.98 \mathrm{eV}$, due to ionization from the ether oxygen lone pair orbital, and a second band at $10.9 \mathrm{eV}$, due to

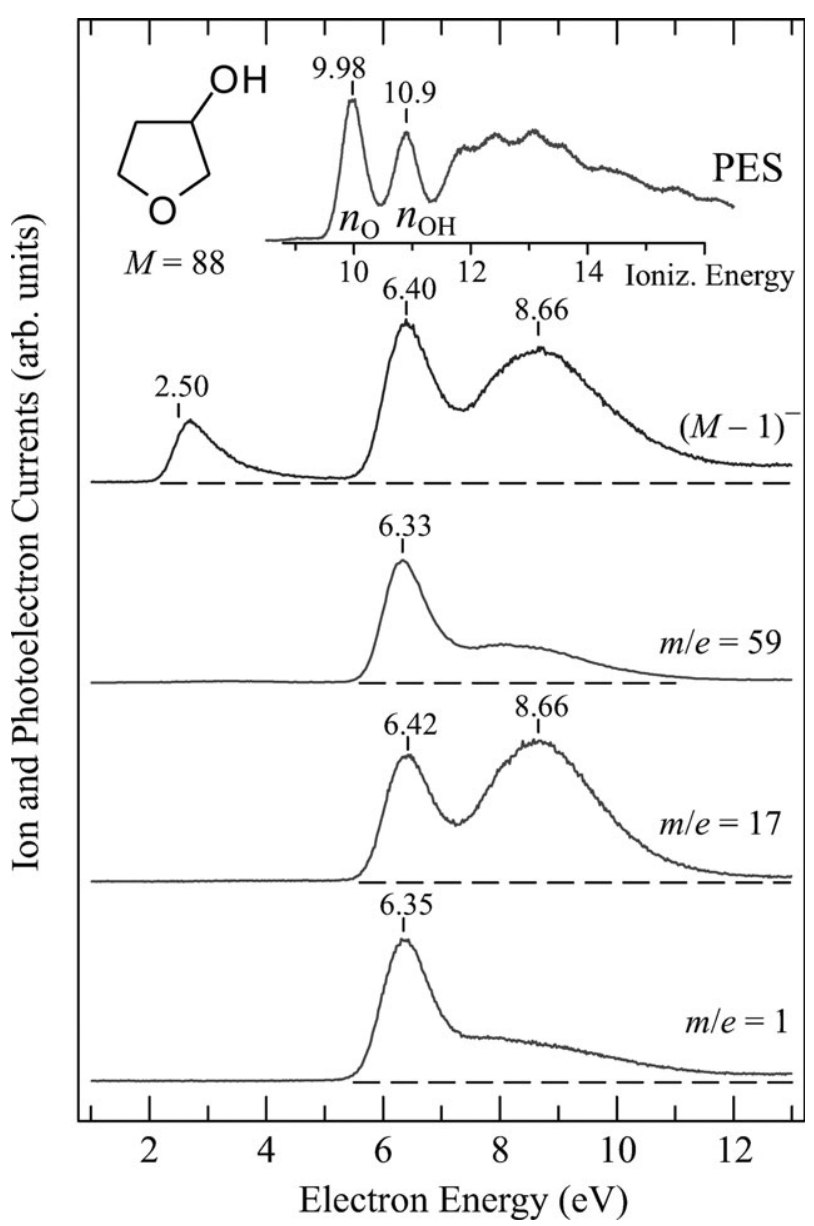

Fig. 9 Photoelectron and DEA spectra of tetrahydrofuran-3-ol (4). The PE spectrum is shifted by $-4.5 \mathrm{eV}$.

ionization from the hydroxyl oxygen lone pair orbital. When the PES band is shifted by an amount similar to the other alcohols, the first DEA band at $6.4 \mathrm{eV}$ appears to correspond to the second photoelectron band, that is, to a Feshbach resonance with a hole on the hydroxyl oxygen. The absolute DEA cross-section of $\mathbf{4}$ (fragments of all masses combined) has been measured by Aflatooni et al. ${ }^{39}$ who obtained a substantial value of $4.9 \times 10^{-19} \mathrm{~cm}^{2}$ at the peak of the $6.2 \mathrm{eV}$ band-in contrast to the parent tetrahydrofuran with a peak crosssection of only $0.16 \times 10^{-19} \mathrm{~cm}^{2}$. The $\mathrm{H}^{-}$yield in Fig. 9 can be compared to the $\mathrm{H}^{-}$yield obtained from 10 monolayer thick film of frozen tetrahydrofuran-3-ol by Antic et al. ${ }^{54,55}$ They observed a shoulder at $7.3 \mathrm{eV}$ followed by a peak at 10 $\mathrm{eV}$, features which probably correspond to the present features at 6.4 and $8.1 \mathrm{eV}$, but shifted toward higher energies by the surrounding molecules in the condensed phase - an effect similar to the blue shift of Rydberg states of neutral molecules. ${ }^{56}$

A band with an onset at $2.50 \mathrm{eV}$ is observed in the $(M-1)^{-}$ spectrum and is assigned to dissociation via a $\sigma_{\mathrm{O}-\mathrm{H}}^{*}$ shape resonance as in the preceding alcohols. The onset is at a distinctly lower energy than in methanol, and this change is reflected in the calculated threshold energy (Table 1). The intensity of this band is substantially larger than that for the alcohols discussed so far. This could be a consequence of the 
stabilization of the $\sigma^{*}$ orbitals due to the presence of two electronegative atoms and to the lower energy of the threshold. The broad band at $8.66 \mathrm{eV}$ is presumably due to Feshbach resonances associated with ionization from the $\sigma_{\mathrm{C}-\mathrm{C}}$ and $\sigma_{\mathrm{C}-\mathrm{H}}$ orbitals.

The fragment with $m / z=59$ whose spectrum is shown in Fig. 9 corresponds to the loss of an ethene molecule from the $(M-1)^{-}$anion. The structure of the $m / z=59$ anion could be either the $\mathrm{OCH}_{2} \mathrm{CHO}^{-}$anion obtained when ethene is split off from tetrahydrofuran-3-ol, or its more stable rearranged form, the acetate anion $\mathrm{CH}_{3} \mathrm{COO}^{-}$. Also observed, but not shown in Fig. 9, was a fragment with $m / z=57$, which corresponds to the loss of a formaldehyde molecule from the $(M-1)^{-}$anion. Its structure could be that of an $\alpha$-deprotonated acetone, isoelectronic with the acetate anion and also stabilized by the delocalization of the charge over three atoms. A fragment with $m / z=41$, probably deprotonated ketene, is observed with an incident energy of $8 \mathrm{eV}$. $\mathrm{OH}^{-}$is presumably the result of direct breaking of the $\mathrm{C}-\mathrm{OH}$ bond.

\section{5 (Tetrahydrofuran-3-yl)methanol (5)}

The spectrum in Fig. 10 reveals that the $(M-1)^{-}$fragment is observed with an onset at $2.36 \mathrm{eV}$, consistent with the calculated threshold (Table 1). Like in tetrahydrofuran-3-ol, the

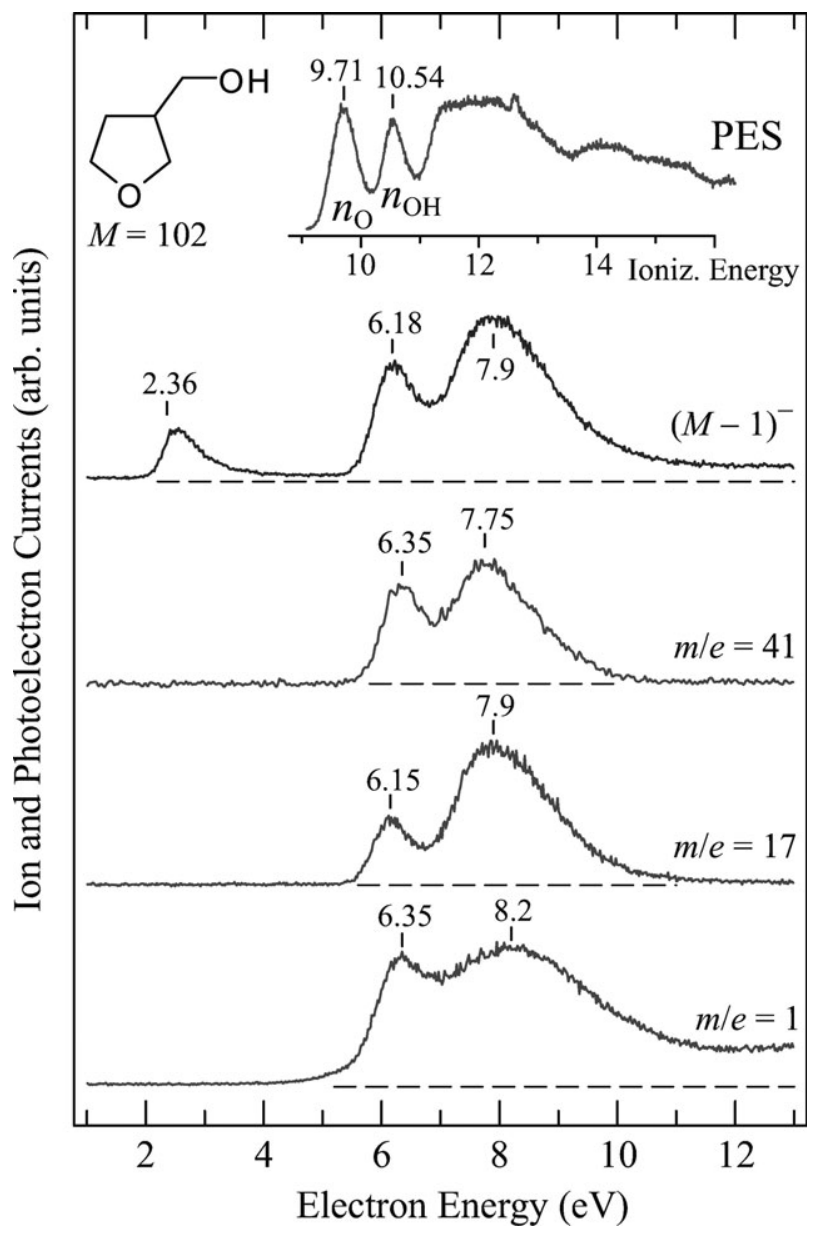

Fig. 10 DEA and PE spectra of (tetrahydrofuran-3-yl)methanol (5). The PE spectrum is shown shifted by $-4.35 \mathrm{eV}$.
PE-spectrum has two low-lying bands, corresponding to ionization from the ether and the hydroxyl oxygen lone pair orbitals, but only one Feshbach resonance band in the 6-7 $\mathrm{eV}$ range. The assignment of the $6.4 \mathrm{eV}$ DEA band to the second PE band appears more probable - the assignment to the first band would require shifting the PE-spectrum by an amount too different from that used for the monoalcohols 1-3. The dominant fragments are $(M-1)^{-}$and $\mathrm{H}^{-}$; the $m / z=41$ and $\mathrm{OH}^{-}$signals are about $5 \times$ weaker than the $(M-1)^{-}$ signal. Like in the previous section, $\mathrm{OH}^{-}$is presumably the result of direct breaking of the $\mathrm{C}-\mathrm{OH}$ bond. The fragment with $m / z=41$ is presumably deprotonated ketene and results from secondary fragmentation of the $(M-1)^{-}$ion.

\section{6 (Tetrahydrofuran-2-yl)methanol (6)}

This compound is structurally closely related to the previous one, with the difference that it can build an intramolecular hydrogen bond, not possible in (tetrahydrofuran-3-yl)methanol. This hydrogen bridge slightly stabilizes the neutral target molecule and has the consequence of slightly increasing the threshold for the $(M-1)^{-}$formation. This slight increase is reflected both in the increase of the energy of the $(M-1)^{-}$ fragment onset (Fig. 10 and 11) and the calculated threshold energy (Table 1).

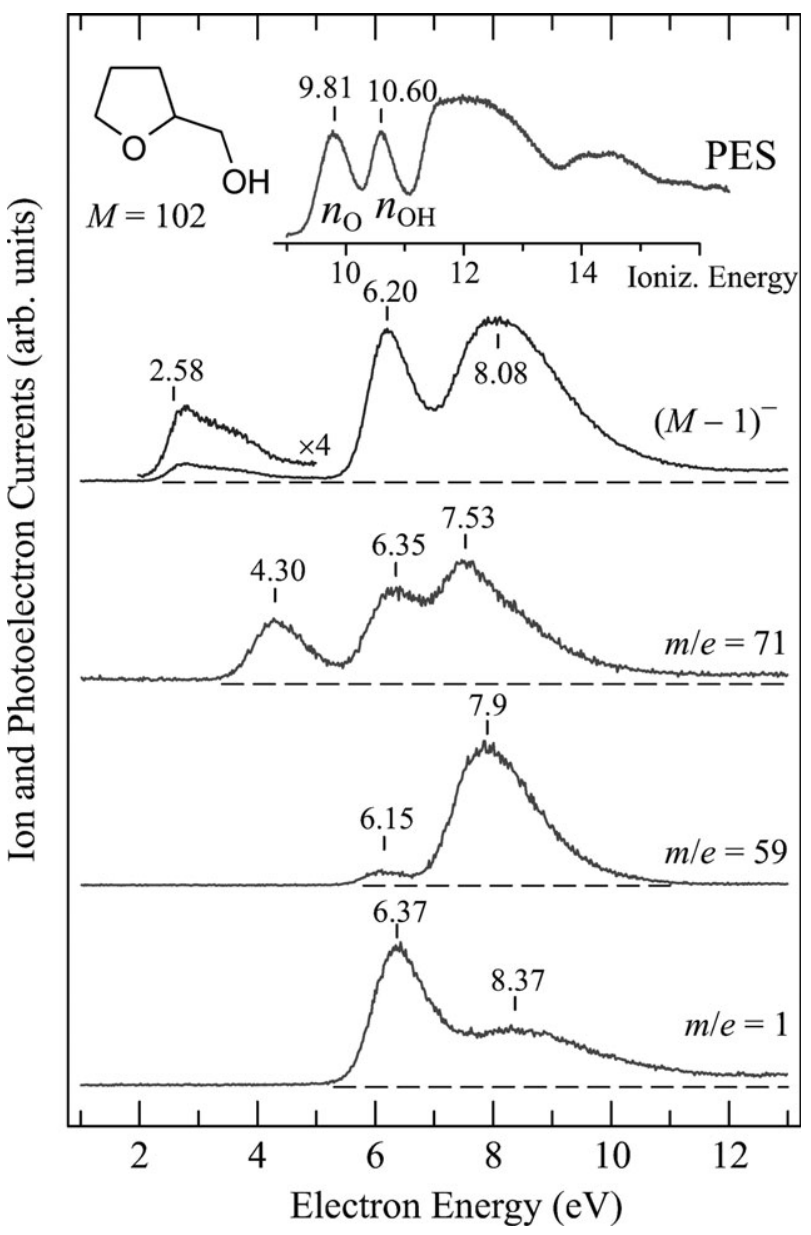

Fig. 11 DEA and PE spectra of (tetrahydrofuran-2-yl)methanol (6). The PE spectrum is shown shifted by $-4.5 \mathrm{eV}$. 
The fragmentation pattern (Fig. 11) in the Feshbach resonance range closely resembles that of the preceding compound (5), with dominant $(M-1)^{-}$and $\mathrm{H}^{-}$fragments, a weaker $\mathrm{OH}^{-}$fragment (not shown in Fig. 11), and spectra of similar shapes. New are the fragments with masses 59 and 71 . The $\mathrm{m} / \mathrm{z}$ $=59$ fragment could correspond to the loss of the ketene molecule from $(M-1)^{-}$and have the propanolate anion structure $\mathrm{C}_{3} \mathrm{H}_{7} \mathrm{O}^{-}$. The $m / z=71$ fragment is exceptional in the present work in being the only fragment other than $(M-$ $1)^{-}$observed already at energies below the Feshbach resonances, with a band peaking at $4.3 \mathrm{eV}$ in Fig. 11. We propose two mechanisms for its formation: either it could correspond to a simple breaking of a $\mathrm{C}-\mathrm{C}$ bond to give the $\mathrm{CH}_{2} \mathrm{OH}+$ $c-\mathrm{C}_{4} \mathrm{H}_{7} \mathrm{O}^{-}$fragments with a calculated threshold at $3.43 \mathrm{eV}$ (Table 2) or it could correspond to the loss of the ethane molecule, $\mathrm{CH}_{3} \mathrm{CH}_{3}$, and have the structure of the conjugate base of the acrylic acid, $\mathrm{CH}_{2}=\mathrm{CHCOO}^{-}$. The latter process has a surprisingly low calculated threshold of $0.42 \mathrm{eV}$ (Table 2 ), but would require a more substantial rearrangement of atoms during the reaction. The onset of this band is above the threshold for both possible explanations, indicating activation energy in the fragmentation. An $m / z=83$ fragment is weakly observed (but not shown in Fig. 11) and could correspond to the loss of a water molecule from the $(M-1)^{-}$anion and have the structure of an $\alpha$-deprotonated cyclopentanone.

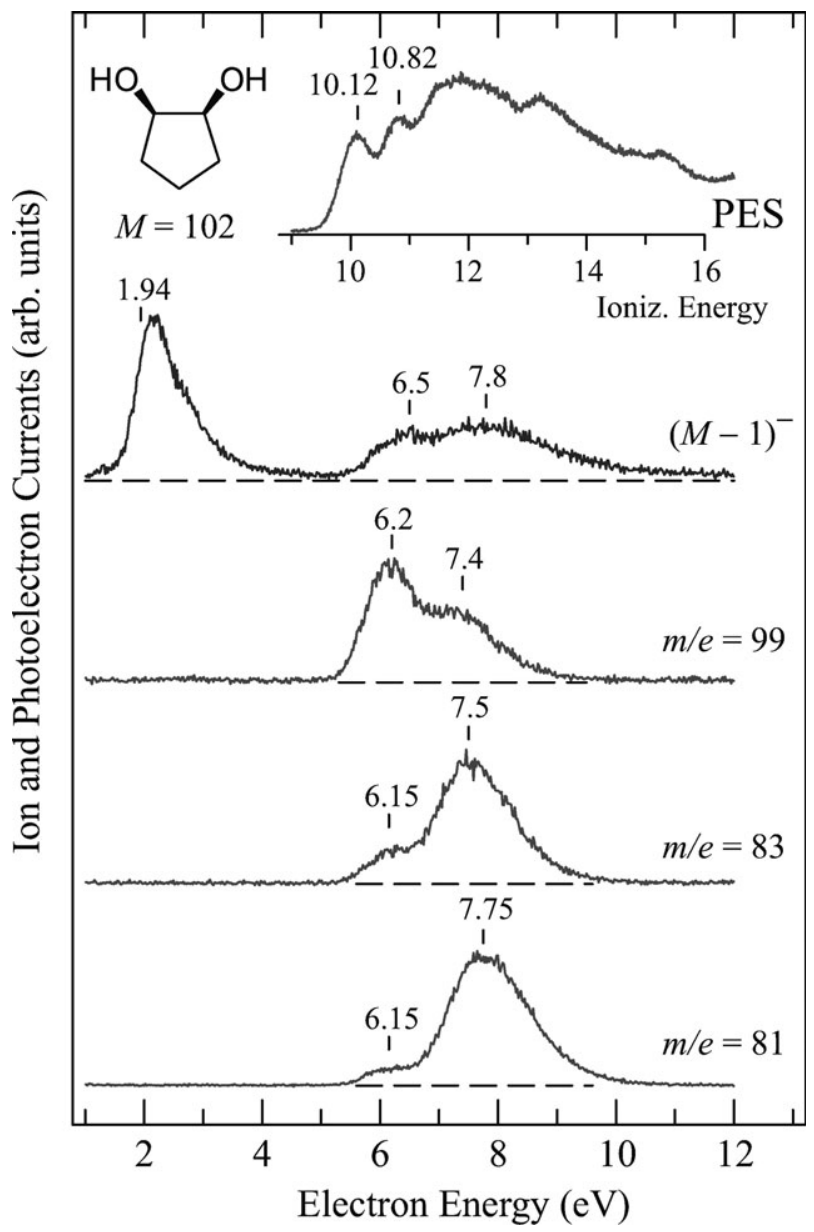

Fig. 12 DEA spectra and the HeI photoelectron spectrum (shown shifted by $-4.5 \mathrm{eV}$ ) of cis-cyclopentane-1,2-diol (7).

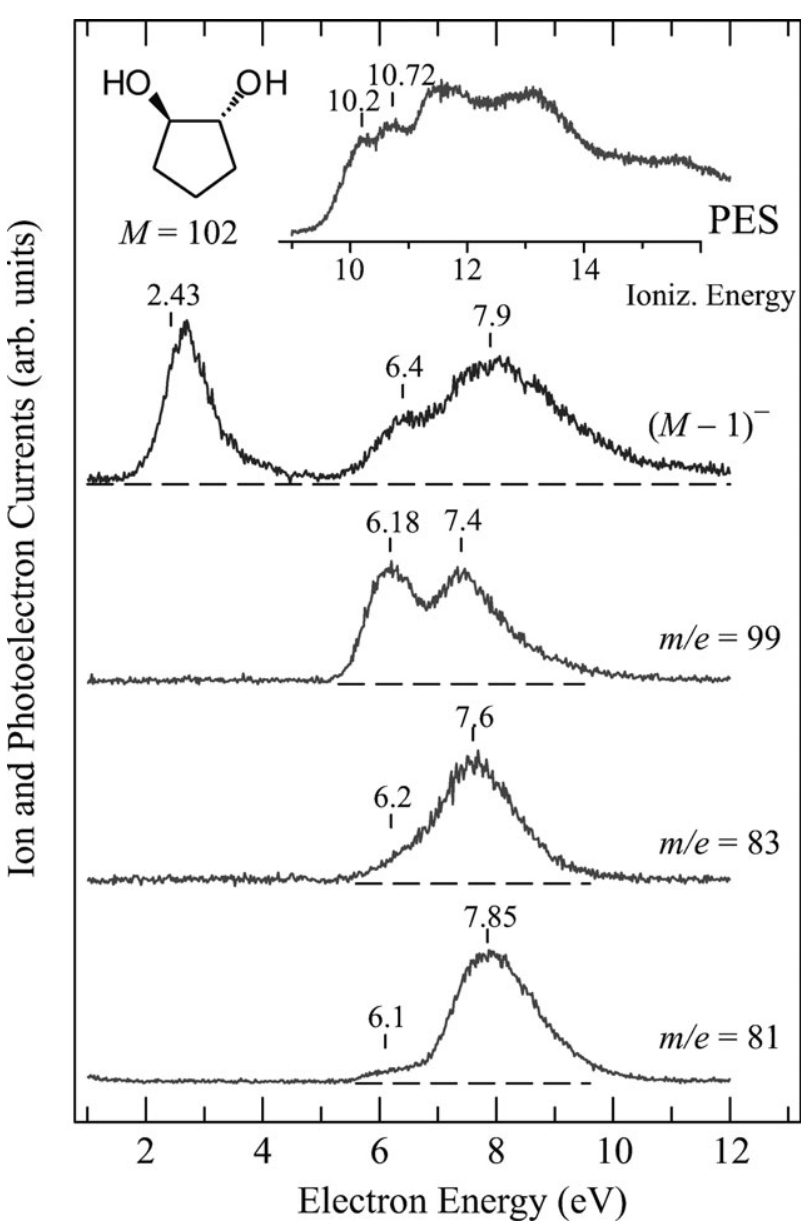

Fig. 13 DEA spectra and the HeI photoelectron spectrum (shown shifted by $-4.5 \mathrm{eV})$ of trans-cyclopentane-1,2-diol (8).

The $\mathrm{H}^{-}$yield in Fig. 11 can be compared to the $\mathrm{H}^{-}$yield obtained from 10 monolayer thick film of frozen (tetrahydrofuran-2-yl)methanol by Antic et al.,,${ }^{54,55}$ with the same conclusions as reached for tetrahydrofuran-3-ol in section 3.4.

\subsection{Cis- and trans-cyclopentane-1,2-diols (7) and (8)}

Fig. 12 and 13 show the PES and DEA spectra of two representative diols. The oxygen lone pair orbitals give rise to two bands in the photoelectron spectrum. MO calculations indicate that charge in the cis compound cation is localized to a large degree - the HOMO is essentially the lone pair on the $\mathrm{O}$-atom participating in a hydrogen bridge whereas HOMO-1 is essentially the lone pair on the $\mathrm{O}$-atom not participating in a hydrogen bridge. The $\mathrm{OH}$ groups in the trans compound are not involved in hydrogen bonds and are thus essentially equivalent. As a consequence, both HOMO and HOMO-1 are delocalized, with equal coefficients on each oxygen lone pair. The two lone pair orbitals in the trans compound are too far apart for a through-space interaction, but both are subject to destabilizing through-bond interactions. MO calculations indicate that the through-bond interaction is slightly stronger for the in-phase combination $\left(n_{\mathrm{O}}+n_{\mathrm{O}^{\prime}}\right)$, which then becomes the HOMO, than for the out-of-phase combination $\left(n_{\mathrm{O}}-n_{\mathrm{O}^{\prime}}\right)$, which becomes the HOMO-1. The consequence is the 'reverse order' of the cationic states. ${ }^{57}$ 
The most striking feature of the DEA spectra of the diols is the decreasing energy and increasing relative intensity of the low energy band in comparison to the monoalcohols - they are more intense than the Feshbach resonance bands.

The decreasing thresholds are the result of the increased electron affinity of the fragments, particularly for the cis compound where the negative charge is stabilized by a hydrogen bridge. The increase in intensity could be a consequence of decreasing autodetachment width of the lowest $\sigma^{*}$ resonance as a result of its decreased energy.

These results are potentially interesting in relation with the DEA spectra of deoxyribose, recently studied by Ptasińska et $a l .{ }^{58}$ and of D-ribose, studied by Bald et al. ${ }^{9}$ Both have a fivemembered ring in their furanose form and the former has three, the latter four hydroxyl groups and may thus be considered as a continuation of the present series of monoand dialcohols. Both in the deoxyribose and the D-ribose intense signals were observed already at $0 \mathrm{eV}$, in continuation of the trend of increasing intensity of the low energy signals with increasing number of hydroxyl groups reported for mono- and dialcohols in this work. New in the sugars are fragmentation channels with very low thresholds, leading to several stable molecules like $\mathrm{H}_{2} \mathrm{O}$ and $\mathrm{CO}_{2}, 9,40,58$ which we did not observe in the alcohols studied here.

\section{Conclusions}

We observe two groups of electron-induced dissociations in hydroxyl-containing compounds. At higher energies, in the 5-10 eV range, the chemistry already well-known from smaller alcohols proceeds via Feshbach resonances, with a grandparent positive ion core and two electrons in diffuse Rydberg-like orbitals.

Additionally to the dissociations mediated by the Feshbach resonances, we report processes at lower energies, $2-3 \mathrm{eV}$, which start at their energetic thresholds. Dramatic H/D isotope effects indicate large autodetachment widths of the lowenergy resonances, which we assign as shape resonances with temporary occupation of $\sigma_{\mathrm{O}-\mathrm{H}}^{*}$ valence orbitals. The corresponding potential surfaces are likely to acquire a dipolebound character and to bend down at intermediate $\mathrm{O}-\mathrm{H}$ distances, similarly to the halogen halide cases.

The importance of the low energy process increases when a second oxygen atom is incorporated in the targets - in the tetrahydrofuran derived molecules - and increases even more when the number of hydroxyl groups is increased from one to two. For the diols the low-energy signal increases when the hydroxyl groups are closer together, that is, when the interaction of the $\sigma_{\mathrm{O}-\mathrm{H}}^{*}$ orbitals of the individual $\mathrm{OH}$ groups is increased, therefore increasing the splitting of the two resulting molecular orbitals and stabilizing of the lower $\sigma_{\mathrm{O}-\mathrm{H}}^{*}$ orbital. The trend toward larger intensity of the low-energy processes appears to be continued and further enhanced by the availability of fragmentations with very low thresholds in the sugars (leading to stable molecules like $\mathrm{H}_{2} \mathrm{O}$ and $\mathrm{CO}_{2}$ ), which have even more $\mathrm{OH}$ groups, and where intense signals have already been observed starting at $0 \mathrm{eV}$. $9,40,58$

Our observations provide indirect insight into the dynamics of the dissociation of the Feshbach resonances - which is not well understood in polyatomic molecules, with the notable exception of the recent detailed theoretical work on $\mathrm{H}_{2} \mathrm{O}{ }^{23}$ The mass spectra do not, of course, give indication of the structure of the fragments, but the present work provides indirect evidence by 'chemical' means, by observing the trends in a number of related compounds. The observations made with the present compounds are that:

- The $(M-1)^{-}$and $\mathrm{H}^{-}$fragments are observed in all cases and generally represent the strongest signals.

- These fragments often carry a 'signature' of the Feshbach resonance involved, hydroxyl hydrogens depart from Feshbach resonances with a hole in the oxygen lone pair orbital, alkyl hydrogens depart from Feshbach resonances with a hole in the $\mathrm{C}-\mathrm{H}$ localized orbitals.

- Fragments other than $(M-1)^{-}$and $\mathrm{H}^{-}$can in most cases be rationalized by subsequent dissociations of the initial $(M-1)^{-}$ion into stable structures, generally closed-shell molecules and closed-shell ions, often further stabilized by charge delocalization. These structures are often quite unrelated to the original structure of the target molecule, substantial reorganization of the atoms is required.

These observations can be rationalized by assuming that the primary step in the dissociation of the Feshbach resonances associated with the ionization of the hydroxyl oxygen lone electrons is the loss of the hydroxyl hydrogen, that is, the acidic proton departs, together with the 'extra' electron. For the Feshbach resonances around $8 \mathrm{eV}$ the primary process may be the loss of an alkyl hydrogen. These processes are, because of the low mass of the proton, fast enough to compete with the fast autodetachment of the resonance-this step is 'kinetically driven', on a time scale comparable to or shorter than a classical vibrational period.

The indirect information on the structures of fragments other than $(M-1)^{-}$and $\mathrm{H}^{-}$indicates that their formation is 'thermodynamically driven', with preference for stable fragments. This indicates that these fragments are formed in a slower secondary reaction from the $(M-1)^{-}$ion. Some of the $(M-1)^{-}$ions formed in the initial fast step are likely to have enough internal energy for the secondary fragmentations. The energy distribution between the kinetic energy of the departing $\mathrm{H}$-atom and the internal degrees of freedom in the initial fast step is to some degree statistical, that is, the $(M-1)^{-}$ion is generated with a distribution of internal energies. (The energy distribution of the departing $\mathrm{H}^{-}$ions has been measured for methanol ${ }^{42}$ and a significant release of kinetic energy has been observed.) At least some of the ( $M-$ $1)^{-}$ions are thus generated hot enough to further dissociate thermally, with preference being given to the most stable fragments, closed shell molecules and ions, which 'boil off' from the primary $(M-1)^{-}$ion. $(M-1)^{-}$ions which either do not have enough energy to dissociate or where the dissociation takes longer than the passage time through the ion lens and the quadrupole mass filter (about $10 \mu \mathrm{s}$ ) are detected with the $M-1$ mass.

An important difference between small molecules (like $\mathrm{H}_{2} \mathrm{O}$ ) and the larger molecules is that the latter have many low-lying thresholds corresponding to the loss of stable molecules like ethene, formaldehyde, ketene, water, etc., opening many more fragmentation channels at relatively low energies. 
In some cases a second fast primary dissociation appears to be the breaking of a $\mathrm{C}-\mathrm{O}$ bond leading to loss of $\mathrm{OH}^{-}$ or $\mathrm{RO}^{-}$.

The low-energy $(2-3 \mathrm{eV})$ processes are limited to the fast dissociations leading to the $(M-1)^{-}$ions, without subsequent fragmentations, although calculations indicate that the thresholds for some of the more complex fragmentations would be low enough. It seems that the primary $(M-1)^{-}$ions do not have enough energy to overcome activation energies involved in the atom 'scrambling' required for the 'thermodynamically driven' secondary fragmentations.

These findings indicate that care must be taken in porting the results from isolated gas phase molecules to condensed phase, where the initially formed hot ion may be rapidly cooled by the surroundings, quenching further dissociations.

\section{Acknowledgements}

This research is part of project No. 200020-113599/1 of the Swiss National Science Foundation.

\section{References}

1 B. Boudaiffa, P. Cloutier, D. Hunting, M. A. Huels and L. Sanche, Science, 2000, 287, 1658.

2 F. Martin, P. D. Burrow, Z. Cai, P. Cloutier, D. Hunting and L. Sanche, Phys. Rev. Lett., 2004, 93, 068101.

3 U. Schmelmer, R. Jordan, W. Geyer, W. Eck, A. Gölzhäuser, M. Grunze and A. Ulman, Angew. Chem., Int. Ed., 2003, 42, 559.

4 E. Illenbergger and J. Momigny, Gaseous Molecular Ions, Steinkopf Verlag, Darmstadt and Springer Verlag, New York, 1992.

5 G. Hanel, B. Gstir, S. Denifl, P. Scheier, M. Probst, M. Farizon, E. Illenberger and T. D. Märk, Phys. Rev. Lett., 2003, 90, 188104.

6 H. Abdoul-Carime, S. Gohke and E. Illenberger, Phys. Rev. Lett., 2004, 92, 168103.

7 A. M. Scheer, K. Aflatooni, G. A. Gallup and P. D. Burrow, Phys. Rev. Lett., 2004, 92, 068102.

8 C. König, J. Kopyra, I. Bald and E. Illenberger, Phys. Rev. Lett., 2006, 97, 018105

9 I. Bald, J. Kopyra and E. Illenberger, Angew. Chem., Int. Ed., 2006, 45, 4851.

10 V. S. Prabhudesai, A. H. Kelkar, D. Nandi and E. Krishnakumar, Phys. Rev. Lett., 2005, 95, 143202.

11 T. Skalický and M. Allan, J. Phys. B, 2004, 37, 4849.

12 L. G. Christophorou, D. L. McCorkle and A. A. Christodoulides, in Electron-Molecule Interactions and Their Applications, ed. L. G. Christophorou, Academic Press, Orlando, 1984, vol. 1, p. 477.

13 Y. Pariat and M. Allan, Int. J. Mass Spectrom. Ion Processes, 1991, $103,181$.

14 H. Hotop, M.-W. Ruf, M. Allan and I. I. Fabrikant, Adv. At. Mol. Opt. Phys., 2003, 49, 85.

15 A. Schramm, I. I. Fabrikant, J. M. Weber, E. Leber, M. W. Ruf and H. Hotop, J. Phys. B, 1999, 32, 2153.

16 M. Allan and T. Skalický, J. Phys. B, 2003, 36, 3397.

17 M. Stepanović, Y. Pariat and M. Allan, J. Chem. Phys., 1999, 110, 11376.

18 P. D. Burrow, G. A. Gallup, A. M. Scheer, S. Denifl, S. Ptasińska, T. Mark and P. Scheier, J. Chem. Phys., 2006, 124, 124310.

19 M. B. Robin, Higher Excited States of Polyatomic Molecules, Academic Press, Orlando, 1985.

20 K. L. Stricklett and P. D. Burrow, J. Phys. B, 1986, 19, 4241.

21 R. A. Dressler and M. Allan, Chem. Phys. Lett., 1985, 118, 93.

22 R. A. Dressler and M. Allan, J. Electron Spectrosc. Relat. Phenom., 1986, 41, 275.

23 D. J. Haxton, Z. Zhang, H.-D. Meyer, T. N. Rescigno and C. W. McCurdy, Phys. Rev. A, 2004, 69, 062714.

24 H. Estrada, L. S. Cederbaum and W. Domcke, J. Chem. Phys., 1986, 84, 152.
25 S. Feuerbacher, T. Sommerfeld and L. Cederbaum, J. Chem. Phys., 2004, 120, 3201.

26 L. Sanche and G. J. Schulz, Phys. Rev. Lett., 1971, 27, 1333.

27 L. Sanche and G. J. Schulz, Phys. Rev. A, 1972, 5, 1672.

28 L. Sanche and G. J. Schulz, Phys. Rev. A, 1972, 6, 69.

29 L. Sanche and G. J. Schulz, J. Chem. Phys., 1973, 58, 479.

30 F. Gresteau, R. I. Hall, A. Huetz, D. Vichon and J. Mazeau, J. Phys. B, 1979, 12, 2925.

31 D. Spence, J. Phys. B, 1975, 12, 721.

32 F. H. Read, J. Phys. B, 1977, 10, 449.

33 D. Spence, J. Chem. Phys., 1977, 66, 669.

34 R. A. Dressler, M. Allan and M. Tronc, Chem. Phys., 1987, 20, 393.

35 V. I. Khvostenko, A. S. Vorob'yov and O. G. Khvostenko, J. Phys. B, 1990, 23, 1975.

36 R. Dressler and M. Allan, Chem. Phys., 1985, 92, 449.

37 M. Allan, J. Phys. B, 1992, 25, 1559.

38 M. J. Frisch, G. W. Trucks, H. B. Schlegel, G. E. Scuseria, M. A. Robb, J. R. Cheeseman, J. A. Montgomery, Jr., T. Vreven, K. N. Kudin, J. C. Burant, J. M. Millam, S. S. Iyengar, J. Tomasi, V. Barone, B. Mennucci, M. Cossi, G. Scalmani, N. Rega, G. A. Petersson, H. Nakatsuji, M. Hada, M. Ehara, K. Toyota, R. Fukuda, J. Hasegawa, M. Ishida, T. Nakajima, Y. Honda, O. Kitao, H. Nakai, M. Klene, X. Li, J. E. Knox, H. P. Hratchian, J. B. Cross, V. Bakken, C. Adamo, J. Jaramillo, R. Gomperts, R. E. Stratmann, O. Yazyev, A. J. Austin, R. Cammi, C. Pomelli, J. Ochterski, P. Y. Ayala, K. Morokuma, G. A. Voth, P. Salvador, J. J. Dannenberg, V. G. Zakrzewski, S. Dapprich, A. D. Daniels, M. C. Strain, O. Farkas, D. K. Malick, A. D. Rabuck, K. Raghavachari, J. B. Foresman, J. V. Ortiz, Q. Cui, A. G. Baboul, S. Clifford, J. Cioslowski, B. B. Stefanov, G. Liu, A. Liashenko, P. Piskorz, I. Komaromi, R. L. Martin, D. J. Fox, T. Keith, M. A. Al-Laham, C. Y. Peng, A. Nanayakkara, M. Challacombe, P. M. W. Gill, B. G. Johnson, W. Chen, M. W. Wong, C. Gonzalez and J. A. Pople, GAUSSIAN 03 (Revision C.02), Gaussian, Inc., Wallingford, CT, 2004.

39 A. Aflatooni, A. M. Scheer and P. D. Burrow, J. Chem. Phys., 2006, 125, 054301 .

40 P. Sulzer, S. Ptasińska, F. Zappa, B. Mielewska, A. R. Milosavljević, P. Scheier, T. D. Märk, I. Bald, S. Gohlke, M. A. Huels and E. Illenberger, J. Chem. Phys., 2006, 125, 044304.

41 A. Kühn, H. P. Fenzlaff and E. Illenberger, J. Chem. Phys., 1988, 88, 7453.

42 M. G. Curtis and I. C. Walker, J. Chem. Soc., Faraday Trans., 1992, 88, 2805.

43 NIST Chemistry webbook: http://webbook.nist.gov/chemistry.

44 Y. Pariat and M. Allan, Int. J. Mass Spectrom. Ion Processes, 1991, 103, 181.

45 C. Mündel, M. Berman and W. Domcke, Phys. Rev. A, 1985, 32, 181.

46 G. J. Schulz and R. K. Asundi, Phys. Rev., 1967, 158, 25.

47 G. J. Schulz, Rev. Mod. Phys., 1973, 45, 423.

48 I. C. Walker, A. Stamatović and S. F. Wong, J. Chem. Phys., 1978, 69, 5532.

49 M. Allan, J. Phys. B, 2006, 39, 2939.

50 J. Horáček, M. Ćížek, K. Houfek, P. Kolorenč and W. Domcke, Phys. Rev. A, 2004, 70, 052712.

51 J. Horáček, M. Ćížek, K. Houfek, P. Kolorenč and W. Domcke, Phys. Rev. A, 2006, 73, 022701.

52 M. Cížek, J. Horáček, M. Allan, A.-C. Sergenton, D. Popović, W. Domcke, T. Leininger and F. X. Gadea, Phys. Rev. A, 2001, 63, 062710 .

53 R. Dressler and M. Allan, J. Electron Spectrosc. Relat. Phenom., 1986, 41, 275.

54 D. Antic, L. Parenteau, M. Lepage and L. Sanche, J. Phys. Chem. $B, 1999, \mathbf{1 0 3}, 6611$.

55 L. Parenteau, D. Antic and L. Sanche, J. Phys. Chem. B, 2000, 104, 4711.

56 M. B. Robin, Higher Excited States of Polyatomic Molecules, Academic Press, Orlando, 1985.

57 R. Hoffmann, E. Heilbronner and R. Gleiter, J. Am. Chem. Soc., 1970, 92, 706.

58 S. Ptasińska, S. Denifl, P. Scheier and T. D. Märk, J. Chem. Phys., 2004, 120, 8505 . 